\title{
Numerical Techniques for Optimization of Gait Generation
}

\author{
Rodrigo Matos Carnier* Non-member, Yasutaka Fujimoto* Senior Member
}

(Manuscript received Nov. 25, 2019, revised Oct. 1, 2020)

J-STAGE Advance published date : Dec. 11, 2020

\begin{abstract}
Optimization of robotic bipedal walking widely uses simplified dynamical models to minimize the computational cost and generate fast solutions, but this poses the problem of modeling errors and increased inaccuracy. Moreover, direct methods of optimization are almost always used; however, most are not implemented to satisfy Pontryagin's Principle, which can lead to suboptimal solutions. This study presents the optimization of planar robotic bipedal walking using exact dynamics. We apply pseudospectral optimal control to optimize gait generation using two accuracy requirements: nonlinear multi-degree-of-freedom whole-body dynamics and satisfied conditions of optimality according to Pontryagin's Principle. In order to consistently achieve solvability of optimization in this type of problem, we present a novel methodology consisting of synergic techniques that address well-known numerical difficulties of optimization.
\end{abstract}

Keywords: bipedal walking, optimal control, whole-body dynamics

\section{Introduction}

Robotic bipedal walking is a field of research experiencing what could be called "the pains of adolescence". It is a new field of study but not so new, and it has a degree of development but still presents many problems and challenges that prevent widespread commercial application ${ }^{(1)}$. Even urgent situations that would justify the use of non-commercial stateof-art prototypes cannot rely on current robots, and efforts are being made to achieve this critical point of development ${ }^{(2)}$. Walking suffers from the thankless characteristic of being one of the most basic abilities required from multipurpose humanoid robots, but also one of the most difficult to properly reproduce ${ }^{(3)}$. Humans possess the very challenging ability of moving while underactuating its mechanical structure, with a lot of flexibility in the parameters of movement. They can multitask while walking and have a highly evolved synergy between motor control and mechanical structure (like the semi-passive dynamics of its body, which allows partly underactuated movement, or the many mechanisms of storage and release of energy, like the Achilles tendon or the elasticity of muscles). All this topped with astounding robustness for such a flexible task.

Since its inception, robotic walking has been performed using different control approaches that, so far, have not been integrated into a single one that clearly reproduces all the fundamental properties of walking. Most popular approaches $^{(3)-(5)}$ depend on a constant actuation of all joints in order to precisely control the state of the system, while semi-passive approaches ${ }^{(6)(7)}$ attempt to reproduce the human synergy between mechanical structure and control through proper mechanical design of underactuation. The former

\footnotetext{
* Department of Electrical and Computer Engineering, Yokohama National University

79-5, Tokiwadai, Hodogaya-ku, Yokohama, Kanagawa 2408501, Japan
}

presents flexibility and robustness but lacks energetic efficiency, while the latter inverts the successes and failures. Between the new approaches, there is one not completely explored yet: optimal control ${ }^{(8)-(10)}$. The ability to define precisely what optimality is desired, and to integrate its realization with the dynamical equations of the system, is very powerful. Optimal control makes it easier to reproduce the synergy between mechanical structure and control, since the improvement of a mechanical structure will automatically increase the limits of performance, leaving for the control the only task of achieving optimal performance by application of optimal control methods. We give an example in Carnier and Fujimoto ${ }^{(11)}$, where the competitiveness between humanlike and avianlike biped walkers was investigated by merely extracting the optimal control of both models for different optimization criteria.

The application of optimal control in bipedal walking possesses some challenges. One is the widespread use of simplified dynamical models. The unstable nature of balance in walking requires stabilization in control systems, and the fast rate of change in the states of a walking system requires fast feedback. This has so far discouraged the use of complete, whole-body dynamics in models of walking systems, since the computational cost of evaluation of dynamics increases exponentially with degrees-of-freedom (DOF). In optimization, this problem is increased in orders of magnitude due to big ammounts of iterations. Using simplified models deals with this and generates fast online solutions, but causes modeling errors. Another challenge is the widespread use of direct methods of optimization. Such methods became hugely popular due to the availability of multiple commercial solvers, simplicity of use and easy solvability, but they present less accuracy and may achieve only suboptimal solutions when not implemented to estimate the costates of Pontryagin's Principle ${ }^{(12)}$ (also called adjoint or dual states). Historically, direct methods were believed to be intrinsically less 
accurate than indirect methods ${ }^{(13)(14)}$. The former use Nonlinear Programming (NLP) to converge optimal solutions by approximations, while the latter apply the optimality conditions of Pontryagin's Principle to dualize the system dynamics (i.e. formulate the costate dynamics) and transform it into a Boundary Value Problem (BVP), which is hard to solve but yields accurate solutions (refer to $\mathrm{Rao}^{(15)}$ for a survey on optimal control methods). However, around two decades ago, developments in optimization techniques, computational power and software tools led to the understanding that the smaller accuracy of direct methods is not due to optimization by NLP in itself, but to not properly estimating the costates during optimization $^{(16)(17)}$. In other words, the problem of smaller accuracy turned out to be not in the method, but in not solving costates to enforce Pontryagin's Principle (be it by actually solving them in an indirect method, or by estimating them in a direct method - see Section 3.1 for more details). But since the majority of current commercial solvers that implement $d i$ rect methods do not estimate the costates, their application in complex optimization ends suffering from the old problem.

The points above demonstrate the current trend of sacrificing proper optimization for achieving online solutions robustly, even if suboptimal. Few studies have performed the optimization of gait of bipedal robots using whole-body dynamics, and only one used a method that satisfies Pontryagin's Principle. A number of studies were made for the robot HRP-2 using whole-body dynamics ${ }^{(18)-(20)}$, generating optimized gaits that were validated by experiments, but all studies relied on direct methods that do not estimate costates to enforce Pontryagin's Principle. More recently, $\mathrm{Hu}$ and Mombaur $^{(21)}$ also generated optimal solutions by a direct method for a whole-body model and validated it in the prototype iCub. The only study found so far that satisfied Pontryagin's Principle was made by Rostami and Bessonet ${ }^{(22)}$. They generated the single support phase of a model of planar robot with 6 DOF using an indirect method for optimization and a shooting method for solving the generated BVP. However, their method took around 25 minutes to find a solution.

This work presents the optimization of robotic bipedal walking using whole-body dynamics and pseudospectral optimization to satisfy Pontryagin's Principle and perform proper optimization. Our objective is to increase robustness by removing modeling errors from simplified models, improve the ability to reach truly optimal solutions and decrease computational speed, all towards the goal of achieving accurate online optimal gait generation. To achieve consistent solvability in the optimization of nonlinear multi-degree-offreedom (MDOF) whole-body dynamical systems, we contribute with a novel methodology that systematizes techniques to deal with well-known numerical difficulties in optimization: exponential growth of analytical expressions, numerical conditioning of differential equations and sensitivity of optimization to the initial guess. To present our results, the optimization of gait generation of a planar robot model of $6 \mathrm{DOF}$ is performed. Three criteria of optimization are analyzed: minimization of joint torques, minimization of nonconservative forces and minimization of joint power. Optimal solutions are achieved in about 30 seconds-a big improvement over the previous work satisfying Pontryagin's Principle.
After this Introduction, Section 2 will describe the modeling of robot structure and dynamics of the system. Section 3 will present the formulation of the optimization problem and an in-depth description of the numerical techniques used to generate optimal solutions for our nonlinear MDOF whole-body model. Section 4 will present the implementation of bipedal gait, from parameter definition to the proof testing of our methodology in improving solvability of optimization. Section 5 will show the treatment of simulation data and the analysis of quantities concerning the optimal objectives, comparing optimal and non-optimal solutions. In the end, Section 6 will present the summary and future works.

\section{Dynamics}

The model used for studying the optimization of biped walking is a planar model of robot with 6 DOF. Control of walking is performed only in the sagittal plane. In order to reduce the number of DOF in the formulation of dynamics, the model is treated as a manipulator fixed in the ground (Fig. 2). A model with the ankle of the support foot fixed to the floor can be considered equivalent to a free floating robot model, as long as the forces and torques applied by the floor to the fixed foot never pull it to the floor, nor take the Zero-Moment Point ${ }^{(5)}$ away from the base of support of the robot.

2.1 Equation of Motion The system states are modelled as the absolute angular position and the angular velocity of the robot links. The position is represented by $\theta$ and is measured in respect to the horizontal axis $(x)$ of the world reference, fixed outside the robot, as can be seen by Fig. 2 . Note that usually robot dynamics is formulated using angles of joints - therefore relative angles-but in our study we use the absolute angles of links. This was done in order to simplify the design of state trajectories and to clarify the analysis of behavior of optimized trajectories (see Section 4.1 for more details).

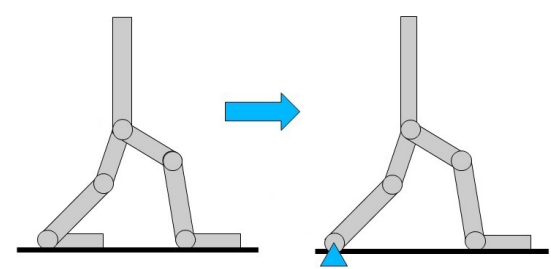

Fig. 1. Modeling of walking robot as a manipulator fixed at the ankle

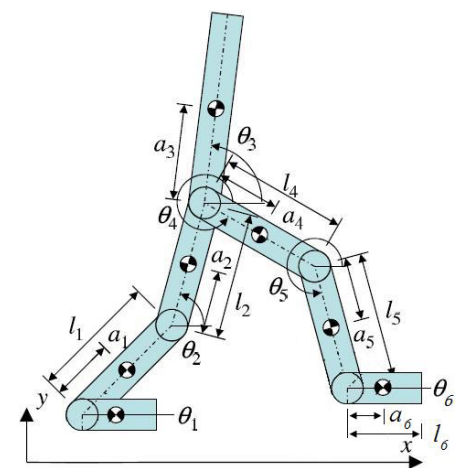

Fig. 2. Planar model with 6 DOF, with representation of link angles 
The dynamics of the mechanical system is derived in the usual way from its Euler-Lagrange Equations ${ }^{(23)}$, resulting in Eq. (1):

$$
M(\theta) \ddot{\theta}+B(\theta, \dot{\theta})+G(\theta)+C(\dot{\theta})=\eta+J^{T} R \ldots \ldots
$$

where $M$ is the Total Inertia matrix, $B$ is the Coriolis and centrifugal effects, $G$ is the gravitational effect, $C$ is the friction acting on joints, $\eta$ is the internal torques working on the links by joint actuators, $J$ is the jacobian of the external forces applied on the end-effector by contact with the ground, and $R$ is the ground reaction forces.

If we group the two vectors of states-angle of links $(\theta)$ and angular velocity of links $(\dot{\theta})$ - in a single vector of states $x(t)$

$$
x(t)=\left[\begin{array}{c}
\theta \\
\dot{\theta}
\end{array}\right]=\left(\theta_{1}, \theta_{2}, \dot{\theta_{1}}, \dot{\theta_{2}}\right)^{T} .
$$

we obtain the following concise formulation of the system dynamics in state-space:

$$
f(x(t), u(t))=\dot{x}(t)=\left[\begin{array}{c}
\dot{\theta} \\
M^{-1}\left(\eta+J^{T} R-B-G-C\right)
\end{array}\right]
$$

The unilateral constraints generated by ground reaction forces $(R)$ are modelled as four elements: $R=$ $\left(R_{x}^{h}, R_{z}^{h}, R_{x}^{t}, R_{z}^{t}\right)^{T}$. The first two elements are the friction and normal forces applied on the first contact point (heel of the swinging foot), and the last two are the friction and normal forces applied on the second contact point (tip of the same foot). They are modeled as Lagrange multipliers and solved in Eqs. (7) and (9). The jacobian of constraints $(J)$ is derived from the function of constraints $g(\theta)$ in the usual way by $J_{i j}=\frac{\partial g_{i}}{\partial \theta_{j}}$. Function $g(\theta)$ bounds the position of the heel and of the tip of foot according to Eqs. (4) and (5):

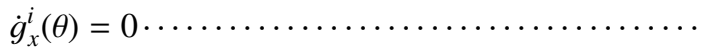

$$
\begin{aligned}
& g_{z}^{i}(\theta) \geq 0
\end{aligned}
$$

where the indexes $x$ and $z$ refer to the horizontal and vertical axis. Eqs. (4) and (5) only apply when there is contact with the ground, which happens during double support phase.

2.2 Constrained Inverse Dynamics The inverse dynamics problem is the evaluation of the inputs necessary to generate a desired rate of change of states, and it is used in our optimization of gait generation to generate an intuitive, non-optimal solution of walking to be used as initial guess for the optimization process. Reformulating Eq. (1) to isolate the torque inputs, we find Eq. (6):

$$
\left[\begin{array}{ll}
J^{T} & I_{6}
\end{array}\right]\left[\begin{array}{l}
R \\
\eta
\end{array}\right]=M \ddot{\theta}+B+G+C \cdots \cdots \cdots \cdots \cdots \cdots
$$

where $I_{6}$ is the $6 \times 6$ identity matrix.

Matrix $\left[\begin{array}{ll}J^{T} & I_{6}\end{array}\right]$ is not square and creates a system of equations that has no unique solution. But inverting this matrix using the Moore-Penrose Pseudoinverse definition ${ }^{(24)}$, and multiplying the right side by it, will yield a best fit solution for $R$ and $\eta$. "Best fit" is meant here as the minimum norm solution of the system, which is the solution that we want since excessive internal forces would only be counterbalanced by ground reaction forces. Eq. (6) is then reformulated as follows, where $A^{+}$is the Moore-Penrose Pseudoinverse:

$$
\left[\begin{array}{c}
R \\
\eta
\end{array}\right]=A^{+}(M \ddot{\theta}+B+G+C)
$$

2.3 Constrained Forward Dynamics The forward dynamics problem is the evaluation of rate of change of states when the inputs are given, and is used by the optimal control solver for two tasks: evaluation the system dynamics during each iteration of optimization and calculation of the control Hamiltonian (Eq. (10)). In this model, this rate of change consists of the angular acceleration of link angles generated by torques acting on the links, and is isolated from Eq. (1) into Eq. (8):

$$
\ddot{\theta}=M^{-1}\left(\eta+J^{T} R-B-G-C\right)
$$

As in the case of constrained inverse dynamics, the evaluation of constrained forward dynamics depends on the simultaneous determination of the desired variables and of ground reaction forces. But unlike the constrained inverse dynamics, the system of equations that solves both accelerations $\ddot{\theta}$ and $R$ can be formulated as a determined system, due to the additional equations provided by path constraints. These equations present another relationship between accelerations and system states, found by taking the second derivative of the constraint equations $g$, giving $-J \ddot{\theta}=\dot{J} \dot{\theta}$. Isolating $\ddot{\theta}$ and $R$ together as the desired variables, we formulate the Eq. (9). The extended mass matrix of the constrained forward dynamics (leftmost matrix) is square and can be inverted normally.

$$
\left[\begin{array}{cc}
M & -J^{T} \\
-J & 0_{4 x 4}
\end{array}\right]\left[\begin{array}{l}
\ddot{\theta} \\
R
\end{array}\right]=\left[\begin{array}{c}
\eta-B-G-C \\
j \dot{\theta}
\end{array}\right]
$$

\section{Optimal Control Problem}

In this work we present a systematic methodology to solve consistently the optimization of gait generation. By using whole-body dynamics and properly satisfying Pontryagin's Principle, we also improve the ability to achieve accurate optimal solutions for robotic walking.

To realize those objectives, four factors come into play: model of the system, formulation of dynamics, optimal control method and numerical solving process. Section 2 described the model and the formulation of dynamics (see Carnier and Fujimoto ${ }^{(25)}$ for the impact of a different formulation in the results of optimization). This section will first decribe the optimal control method used, then will present the numerical solving process in terms of a novel methodology, with in-depth description of the numerical techniques and strategies used to achieve consistent solvability and better computational speed than previous related studies.

\subsection{Optimization Satisfying Pontryagin's Principle}

The optimal control method used in this study is a pseudospectral method that satisfies Pontryagin's Principle for proper optimization. This principle revolves around the control Hamiltonian (Eq. (10)), an extension of the cost function that includes the system dynamics. (We use the prefix "control" to distinguish this Hamiltonian from the one of Hamiltonian mechanics.)

$$
H=l+\lambda^{T} f
$$




$$
O=\int_{t_{0}}^{t_{f}} l d t \ldots \ldots \ldots \ldots \ldots \ldots \ldots \ldots \ldots \ldots \ldots \ldots \ldots \ldots \ldots
$$

where $H$ is the control Hamiltonian, $l$ is the running cost, $\lambda$ is the vector of costates, $f$ is the phase-velocity vector, or system dynamics defined previously, and $O$ is the cost function.

The control Hamiltonian possesses important properties condensed in the optimality conditions (Eqs. (12)-(14)). These conditions need to be satisfied for proper optimization. For a problem with fixed initial and final states, Pontryagin's Principle is stated as follows:

Given an cost function, if $u^{*}$ is an optimal control that minimizes this cost function subject to a differential equation representing the system dynamics, and if $x^{*}$ denotes the corresponding optimal state, then there is an optimal costate $\lambda^{*}$ such that:

$$
\begin{aligned}
& \frac{\mathrm{d} H}{\mathrm{~d} u^{*}}=0 \ldots \ldots \ldots \ldots \ldots \ldots \ldots \ldots \ldots \ldots \ldots \ldots \ldots \ldots \ldots \ldots \ldots \ldots \ldots \\
& \frac{\mathrm{d} H}{\mathrm{~d} \lambda^{*}}=\dot{x}^{*}=f \\
& \frac{\mathrm{d} H}{\mathrm{~d} x^{*}}=-\lambda^{*}
\end{aligned}
$$

And for all $t \in\left[t_{i}, t_{f}\right]$ :

$$
H\left(\lambda^{*}, x^{*}, u, t\right) \geq H\left(\lambda^{*}, x^{*}, u^{*}, t\right)
$$

According to the Principle, the relationship between optimal states, optimal costates and the control Hamiltonian guarantees that the Hamiltonian achieves its minimum value over the trajectory when the states and costates are optimal. Eq. (12) is fundamental to this and is used by many methods as the optimization rule. However, the costates $\lambda$ are an important element of optimization too. They are the dualization of the system states in respect to optimization. Here, $\lambda$ are functions that add "optimization constraints" to the system dynamics, much like Lagrange multipliers are constants that add path constraints. Their main characteristic of interest for us is that they "weight" the rate of change of states $(f)$ in order to fulfill the optimization objective. When the control Hamiltonian is calculated, we are evaluating a criterion of optimization that includes a weighted system dynamics. This is one of the reasons why solving (or estimating) the costates is so important for accurate optimization. In the same way that evaluating constrained dynamics without accurately evaluating the constraints may lead to inaccurate solutions, performing optimizations without evaluating the "optimization constraints" may allow unnoticed violations of the optimality conditions of Pontryagin's Principle, thus generating inaccurate solutions.

Most direct methods of optimization do not estimate the costates during optimization to enforce Pontryagin's Principle, leading to smaller accuracy and sometimes suboptimality. In this study we used the solver DIDO, which implements a direct method that does so. It discretizes the system dynamics and performs a curve approximation that satisfies at the same time the trajectory generation and the optimality conditions. The method is a pseudospectral optimal method based on the Stone-Weierstrass Theorem and the Covector Mapping Principle ${ }^{(16)}$. The estimated costates can be used to tune the optimization to satisfy Pontryagin's Principle (see Section 3.3.2 for details). A user guide for DIDO is found in Ross ${ }^{(26)}$. For more details on the pseudospectral optimal control method, refer to Ross and Fahroo ${ }^{(27)}$. For a more indepth explanation of Pontryagin's Principle and its general application to engineering, refer to Ross ${ }^{(8)}$ and Kirk ${ }^{(9)}$.

3.2 Formulation of an Optimal Problem for Walking

To demonstrate the ability of our methodology to solve different objectives of optimization, three optimizations were realized separately: minimization of joint torques, of joint friction (to decrease energy consumption indirectly) and of joint power (to decrease energy consumption directly). Below are the formulation of cost functions for each optimization.

(1) Minimization of Joint Torques: A common objective of optimization in robotics is the generation of smooth controls by minimizing the square of torques applied in the joints. A secondary effect is a small decrease in energy consumption by indirectly affecting the power applied in the joints. To reinforce this effect, the square of joints angular velocities are added to the square of torques inside the cost function, but the weight of each group of terms ( $a$ for torques and $b$ for velocities) is set to favour minimization of torques. The cost function for this case is given by Eq. (16).

$$
\begin{aligned}
l & =\frac{a}{2} \tau^{T} \tau+\frac{b}{2} \dot{q}^{T} \dot{q} \\
& =\frac{a}{2}\left(G^{-1} \eta\right)^{T}\left(G^{-1} \eta\right)+\frac{b}{2}\left(K^{-1} \theta\right)^{T}\left(K^{-1} \theta\right)
\end{aligned}
$$

where $\tau$ are the joint torques and $G$ is a transformation matrix from link torques to joint torques.

(2) Minimization of Joint Friction: In our dynamical model, non-conservative forces are modeled as viscous friction in the joints $(C=\mu \dot{q})$. They are minimized by taking the square of friction (which is proportional to the joint angular velocities) as the objective of optimization. By decreasing the overal angular velocity of the problem, friction loss is directly decreased too. The cost function for this case is given by Eq. (17).

$$
l=\frac{1}{2}(\mu \dot{q})^{T}(\mu \dot{q})=\frac{1}{2}\left(\mu K^{-1} \theta\right)^{T}\left(\mu K^{-1} \theta\right) \cdots \cdots \cdots
$$

where $\dot{q}$ are the joint angular velocities and $K$ is a transformation matrix from link angular velocities to joint angular velocities.

(3) Minimization of Joint Power: To minimize the energy consumption of the system directly, a second criterion of optimization is defined as the minimization of power applied in the joints. Since the term is not squared, it turns the optimization into a non-convex problem, usually a problematic one. The results of this direct minimization of energy consumption is compared with the indirect minimization by decreasing friction losses. The cost function for this case is given by Eq. (18).

$$
l=\tau^{T} \dot{q}=\left(G^{-1} \eta\right)^{T}\left(K^{-1} \theta\right)
$$


To solve these optimizations, DIDO takes the formulation of cost function, control and path constraints, system dynamics, boundaries of search space for states, controls and constraints, and an initial guess. How these formulations and quantities are provided for the solver makes the difference between success and failure of optimization. While optimal control designers develop a deep knowledge based on experience for solving optimization of complex nonlinear systems, they seldom present the details of the solving process in a systematic way. In the following section, we present a novel methodology in order to fulfill this very necessity.

\subsection{A Novel Methodology for Nonliner Whole-body} MDOF Optimization Nonlinear optimization is well known to be hard to solve. To make things worse, humanoid robots have MDOF, which increase the difficult exponentially. In our previous experience of optimization of human walking ${ }^{(28)}$, we used an indirect method to formulate the optimal control problem and achieve accurate optimization, but managed to solve only 2 and 3 DOF models representing legs, due to the increase of numerical conditioning after 3 DOF. To solve the proper optimization of wholebody gait generation of a complete robot model ${ }^{(29)}$, we had to perform smart formulation of dynamics, use conceptually consistent initial guesses and tune the optimization process iteratively by analysis of outputs. The following collection of numerical techniques and their synergic use are the formalization of this experience into a methodology. The techniques were applied using the pseudospectral optimal method implemented in DIDO, but can (and should) be used with any other method/solver to optimize robot gait generation (or other problems with nonlinear MDOF whole-body dynamics). Some techniques are original by us while others are not, but our main contribution is their organization into a methodology for consistent solvability of optimization.

\subsubsection{Dynamic Symmetry, Recursion and Elimination} of Symbolic Expressions The first way to improve the optimization process consists of computational measures to decrease the computational cost: formulate the dynamics in state-space form to isolate the dynamical variables efficiently, avoid evaluating symbolic expressions only at the final dynamical step and exploit the symmetry of the total inertia matrix in the system dynamics ( $M$ in Eq. (1)).

Inertia matrixes are positive semi-definite and therefore symmetrical. Eliminating the evaluation of symmetrical elements cuts the effort of calculating $M$ by roughly half as we increase the DOF. However, the truly important measure is to evaluate the symbolic expression of this matrix before inverting it, since the symbolic expression of $M^{-1}$ of nonlinear systems becomes huge with just a few DOF. $M^{-1}$ is necessary to calculate the system dynamics (Eq. (3)) and will prevent any hope of online application (or even solvability) if evaluated from a symbolic expression. An even more problematic matrix is the derivative of the inverse inertia matrix $\left(d M^{-1} / d t\right)$. While the calculation of the system dynamics does not require this matrix, the calculation of the dynamics of covectors does and needs to be provided by the control designer in indirect methods. Using the identity of Eq. (19) is fundamental to avoid the even huger symbolic expression of $d M^{-1} / d t$, leaving only $M^{-1}$ and $d M / d t$ to be evaluated.

$$
\frac{\partial M^{-1}}{\partial \theta}=-M^{-1} \frac{\partial M}{\partial \theta} M^{-1}
$$

To perform these evaluations in the context of Sections 2.2 and 2.3, symbolic expressions need to be generated and evaluated separately for every dynamical term presented in those sections $(M, B, G, C, J$ and $\eta$ or $\theta)$. Then their values are used in simple numerical operations to evaluate the system dynamics.

After evaluating the matrix $M$, its numerical value can be inverted and used in any operation, but in operations of the type $a * b=c$, where $a$ is the matrix $M, b$ is the desired vector and $c$ is a given vector (like in our case), it is faster and more accurate numerically to perform a LU factorization of $M$ (i.e., its factorization into two triangular matrixes), then perform forward and back substitution to evaluate $b$.

3.3.2 Scaling and Balancing of Differential Equations

The second technique is related to numerical conditioning, i.e., the sensitivity of numerical processes to the change of its variables. In optimization, there are two aspects of this sensitivity: the numerical conditioning of solving the differential equations of states and the impact of costates in optimization. The former is improved by scaling the units of the states, and the latter by balancing the units of the costates. We will start with scaling, then detail balancing.

Scaling is the change of the units of states in order to make the magnitude of their trajectories assume close values. When the values of trajectories of different states have very different magnitudes, the contribution of each state to the differential equation being solved becomes uneven. Considering how numerical processes perform big amounts of digital calculations and generate small imprecisions by rounding numbers, the impact of states with small magnitudes in the differential equations can be erased by states with big magnitudes. A change of units will even their contributions and can significantly improve the numerical solving process.

This sounds like normalization but is conceptually different. Normalization is the change of units to a canonical base (usually the unitary base), sometimes for easier visualization of a solution or better conceptual understanding of proportion between states. Scaling is the change of units to any base that reduces numerical conditioning of the solving process. Sometimes, scaling will resemble normalization due to the change of magnitudes of trajectories to unitary average values, but Ross et al. ${ }^{(30)}$ demonstrated how this choice of units can sometimes be actually worse than the original units. Also, scaling is an iterative process performed a posteriori until the results of optimization are satisfactory, and not a conceptual change a priori like normalization.

Eqs. (20) and (21) show how to scale a system with 1 DOF, where the unit is changed from $U_{i}=1 \mathrm{~m}$ to $U_{f}=2 \mathrm{~m}$. The variable $x$ represents the state in the old unit, while $\bar{x}$ the state in the new unit. Eq. (21) shows the differential equation $\dot{\bar{x}}$ in the new unit:

Scaling is the part of the technique that affects the states. But while the numerical process can become sensitive to the scaling of states, the optimization process is always very sensitive to the costates, due to their function as "optimization constraints" (as explained in Section 3.1). Therefore, they frequently need to be balanced. 


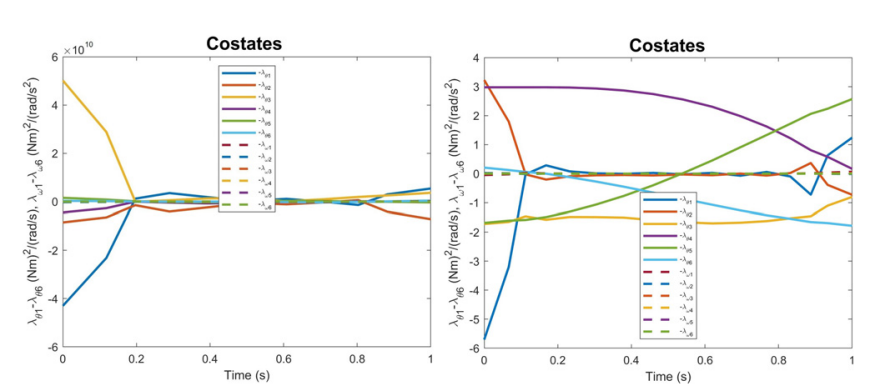

Fig. 3. Examples of different behavior of costates for our 6 DOF system after attempting the same optimization. Left side: failed optimization. Right side: successful optimization

$$
\begin{array}{lll}
U_{i}=(1 m) & & \\
U_{f}=(2 m) & \dot{\bar{x}}=\frac{\dot{x}}{U_{f}} \\
x=1(m) & \dot{\bar{x}}=\frac{f\left(\bar{x} U_{f}\right)}{U_{f}} \\
\bar{x}=0.5(2 m) & \\
x=\bar{x} U_{f} & \\
\dot{x}=f(x)=f\left(\bar{x} U_{f}\right) &
\end{array}
$$

where $U_{i}$ is the initial unit of $x$ and $U_{f}$ is the final unit. For our system with 6 DOF, the scaling of all equations (Eq. (8)) is simply done equation by equation, just like the 1 DOF.

Balancing is an iterative process of checking the behavior of trajectory of costates (mainly if their values are exploding), changing their units, repeating the optimization process and checking again. In other words, because the optimization is sensitive to costates, they can be used as a thermometer of success of optimization and a step of verification/validation. A typical behavior of costate due to failed optimization is given in the left side of Fig. 3, where the trajectory of costates is exploding. The right side of the same figure shows the opposite: "good-behaviored costates" whose trajectories have magnitudes similar or close to the magnitudes of states. In such cases, the optimization is partially validated and can proceed to further verification (e.g. the behavior of the control Hamiltonian in Eq. (12)).

After explaining the motivation of balancing, we now will explain the procedure. Costates have their own dynamics, but depending on the method, either these dynamics are explicitly formulated in differential equations (e.g. (indirect methods)), or the values of costates are estimated without explicit dynamics ((direct methods) $\left.{ }^{(17)}\right)$. If the costate dynamics are formulated explicitly by the control designer, the costates are scaled instead of balanced, by the same method of scaling mentioned above. But if they are estimated by the solver (like DIDO), they need to be "balanced", i.e. scaled indirectly by changing the units of both states and the cost function. This is possible because of the relationship between the unit of these variables, shown in Eq. (22):

$$
U_{\lambda}=\frac{U_{\text {cost }}}{U_{\text {state }}} \ldots \ldots \ldots \ldots \ldots \ldots \ldots \ldots \ldots \ldots \ldots
$$

where $U_{\lambda}$ is the unit of one costate, $U_{\text {cost }}$ is the unit of the cost function and $U_{\text {state }}$ is the unit of the state corresponding to the costate in question.

Scaling of states may affect the optimization, but usually they are scaled just to balance the costates. We stress that all states and costates are interdependent and sensitive to each other, and that overscaling of states will backfire and break the optimization. Therefore the process needs to be tuned iteratively. The procedure is given below:

(1) Solve the OCP and plot the states/costates trajectories in a graphic.

(2) Evaluate the trajectory of the control Hamiltonian (Eq. (10)) and plot it in a graphic.

(3) If the control Hamiltonian is not satisfying Eq. (12), and/or if the values of costates are exploding, the solution is not an optimal solution.

(4) Scale/balance the system by changing the units of states through Eqs. (20) and (21). If the value of a costate is exploding, increase the unit of the correspondent state (the unit of the costate will then decrease). If the control Hamiltonian is not satisfying Eq. (12), try to bring all the magnitudes of states and costates to an approximate average (this will probably take many iterations).

(5) Repeat all steps until the control Hamiltonian and the costates start behaving as expected.

It is recommended to trimm first the difference of order of magnitude of states alone if they are too disparate, then balance the costates until a good trade-off is achieved. Because of the indirect change of units of costates, it is usually not possible to scale/balance both groups of equations completely (i.e. to bring the magnitudes of all trajectories to the range $[0,1])$, but as long as they can be smoothed enough to make the optimization successful, it is sufficient.

As a final observation from experience, we note that the necessity of scaling/balancing depends deeply on the problem being solved, and even different cost functions for the optimization of the same model have different needs. Our optimization of friction (Eq. (17)) did not require scaling at all. The value of it's costates did not explode and the trajectory of the control Hamiltonian was constant as expected by Pontryagin's Principle. However, our optimization of torques (Eq. (16)) required a lengthy iterative process of solving and tuning the scaling/balancing until a proper solution was found. The reason was because the trajectories of three costates had magnitudes around three orders of magnitude higher than the magnitudes of the states. After they were properly balanced (by changing the unit of the correspondent states), the optimization finally succeeded.

For more examples of this technique, refer to Ross et al. ${ }^{(30)}$ and Elissar ${ }^{(31)}$.

3.3.3 Initial Guess for Numerical Solver based on Problem-specific Parameters Providing a initial guess for the solution is required to initialize the optimization process. Initial guesses can have a huge impact on the ability of finding the global minimum or even of finding a solution, and a good guess will at least speed the solving process. An old strategy to generate one is to use a fast optimization method to create a rough solution, and then use it as the initial guess for the originally intended optimization method, ultimately generating an accurate solution (Ross ${ }^{(16)}$ reports the use of $d i$ rect methods to generate guesses for indirect methods as the 


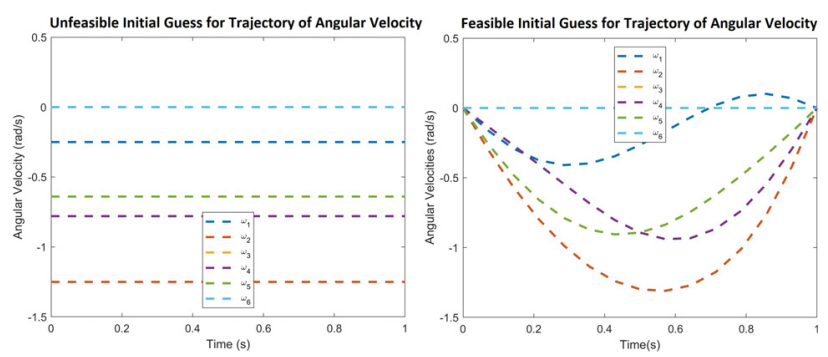

Fig. 4. Examples of bad and good initial guess. Left side: a typical bad guess with constant trajectories, which are not feasible, frequently breaking the optimization. Right side: a feasible initial guess that satisfies the robot dynamics and improves solvability

state-of-art strategy in the mid $90 \mathrm{~s}$ ). But there is another option: to create a feasible non-optimal solution based on any gait generation strategy and then use it as initial guess. A first advantage is that mechanical aplications like the gait of biped walkers can generate such solutions much faster than a first pass of a numerical method. But more importantly, it works because part of the ill-conditioning of nonlinear differential equations comes from the way that numerical methods perform curve fittings and break the feasibility of state trajectories in case of bad guesses. By using a feasible non-optimal solution as initial guess, the method of convergence will already depart from a feasible trajectory and will only adjust it to optimality. This has shown to improve both speed and solvability in our study (see Fig. 4).

Even though using a feasible initial guess is not an uncommon practice, our approach is more specific than choosing any feasible solution. The designer needs to create a feasible solution based on the balancing strategy of gait generation most suited to his objectives. The reason is because different balancing strategies (gaits based on the ZMP concept, or the interted pendulum model, or limit cycles, for example) have different dynamical properties, and some of these are carried over from the initial guess to the optimal solution. The consequence of this is a bigger conceptual control of the designer over the dynamical properties of the optimal solution.

To understand this point, let's remember that sometimes an optimization achieves only a local minimum instead of the global one, and the initial guess has a big impact on this. By choosing a specific balancing strategy for generating the initial guess, and enforcing it during optimization, dynamical properties of the solution are carried over to the optimal solution even if it is only a local minimum. For example, the semi-static ZMP balancing strategy employed by this study is well known to be rather limited in terms of walking speed and size of gait step, since bigger speeds and steps make the robot topple and fall. But since we wanted a more conservative walking, slower but very stable, we choosed this balancing strategy as initial guess and enforced ZMP constraints in the optimization. Even if the optimal solution were a local one, it would still have the dynamical properties of a gait based on the ZMP concept. Besides, the initial guess is also used for our 4th technique: tuning of optimization parameters (see the next Section 3.3.4), which also impacts the solvability. So in many ways the optimization becomes a matter of optimizing a pre-chosen balancing strategy instead of trying to find a global minimum from scratch (e.g. from constant values as initial guess), improving the solvability of fast optimization methods/solvers and increasing the conceptual control over the dynamical properties carried over from the initial guess to the optimal solution. This effectively connects the design of an optimized trajectory to the design of an intuitive nonoptimal solution with desired dynamical properties.

3.3.4 Analysis of Initial Guess for Tuning the Formulation of OCP in the Solver The formulation of the OCP in many solvers requires the definition of search spaces for states, controls and constraints. To perform the optimization, solvers sweep these spaces looking for the solution. The use of bounded search spaces leads to a "global" method that finds the "global" minimum inside the restricted search space. The implementation of search spaces in solvers changes somewhat the logic of initialization from "requiring a initial guess close to the solution" to "requiring search spaces close to the solution". An important consequence is the possibility of improving the solvability of optimization when the solution is not converging, by decreasing the size of the search space. This in turn brings the question of where to put the reduced boundaries. Our approach is to extract these boundaries from the amplitude of the trajectories of the feasible initial guess itself. This will also reinforce the transference of dynamical properties from the initial guess to the optimal solution. This technique-original by us-can also be applied to other solvers by artificial implementation of search spaces.

The extraction of proper search spaces from the initial guess starts with the inspection of the amplitudes of the trajectories of states and controls in the initial guess. Then value of the extremities of each amplitude is set as the lower and upper bounds of the search space of the correspondent state/control. This is done for every state and control. Then these ranges of values are slightly expanded to allow a margin of work for the solver to explore, in order to find the extremal solution without making the solution lose feasibility during the curve approximation. The expanded ranges are then used as search spaces (see Fig. 5).

In case the optimization is performed with a solver that does not limit the search space for states and controls, tuning of the OCP can be made by limiting the search spaces through constraints. This could be done by formulating the constraints as penalty functions and inserting them in the cost function of the system. Since the optimization process will
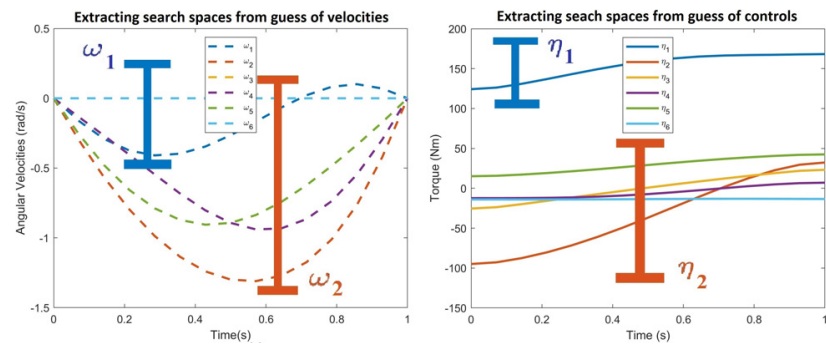

Fig. 5. Example of how to extract search spaces from the amplitudes of two states (left side) and two controls (right side). The extremities of the amplitude are taken as an approximation to the lower and upper boundaries of the search space 
minimize it, the value of the penalty function will also be minimized until it is nullified (which happens when the constraint is not violated anymore). This will enforce the search space for states and/or controls. However, the insertion of many constraints may make the optimization nonsmooth and harder to solve, and care should be taken to not decrease the solvability of the optimization instead of improving it. For more details on this take, refer to Carnier and Fujimoto ${ }^{(28)}$.

\section{Implementation and Results}

In this study, walking is generated as a change of the angular position of every link from an initial state to a final state in a fixed time. The task of walking is divided into two phases:

(1) Single support phase: the end-effector position (the tip of the swinging foot) is brought from a backward position to a forward position.

(2) Double support phase: the end-effector position is kept in the ground where it landed, and the hip of the robot is moved from a backward position to a forward position.

4.1 Generation of Initial Guess As explained in Sections 3.3.3 and 3.3.4, our methodology for the optimization of gait generation requires a feasible solution as initial guess. This solution is generated by applying the constrained inverse dynamics (Equation (7)) of the model on a user-designed trajectory of states. The steps of generation of this initial guess are explained in the sequence.

\section{(1) Define parameters of walking:}

The complete gait cycle has stride of $0.6 \mathrm{~m}$, performed in two phases lasting 1 second each. Initial and final velocities are equal to zero in both phases. The height of the hip is kept constant at $0.5 \mathrm{~m}$ above the ground.

(2) Create trajectories in workspace for swinging foot and trunk:

Trajectories for main translating links are created in workspace: In the single support phase, the swinging foot performs a shallow parabole in the air, while in the double support phase, the torso performs a horizontal line.

\section{(3) Create angular trajectories for each leg independently:}

Trajectories of angular position are created for the 2 top links of each leg (thigh and lower leg) by treating each leg as an independent manipulator with 2 DOF, then solving its geometric inverse kinematics. The inverse kinematics is solved for the trajectory of the manipulator end-effectors: the hip joint and ankle joint. These trajectories are the same as the main translating links, due to the next step.

(4) Create constant angular trajectories for the feet and trunk:

Constant angular trajectories are created for both feet and torso: the attitude of both feet is kept horizontal during all time, while the attitude of torso is kept vertical. If the dynamics were formulated in joint reference, the coupling of joint angles related to these links with constant attitude would be hidden, and their trajectories would need to be solved together with the joints of the legs in a more complex inverse
Table 1. Dimensions and mass of the 6 DOF robot model

\begin{tabular}{|c|c|c|}
\hline LINK & LENGTH & MASS \\
\hline Torso & $0.61 \mathrm{~m}$ & $38.44 \mathrm{~kg}$ \\
\hline Thighs & $0.314 \mathrm{~m}$ & $5.67 \mathrm{~kg}$ \\
\hline Lower legs & $0.425 \mathrm{~m}$ & $2.637 \mathrm{~kg}$ \\
\hline Feet & $0.3 \mathrm{~m}$ & $0.822 \mathrm{~kg}$ \\
\hline
\end{tabular}

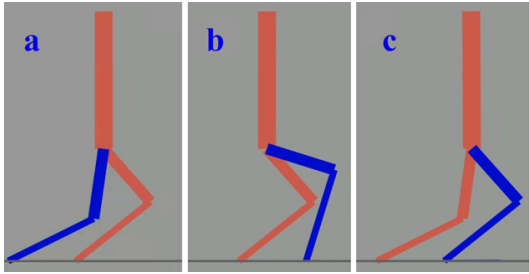

Fig. 6. Robot stances between the phases of walking
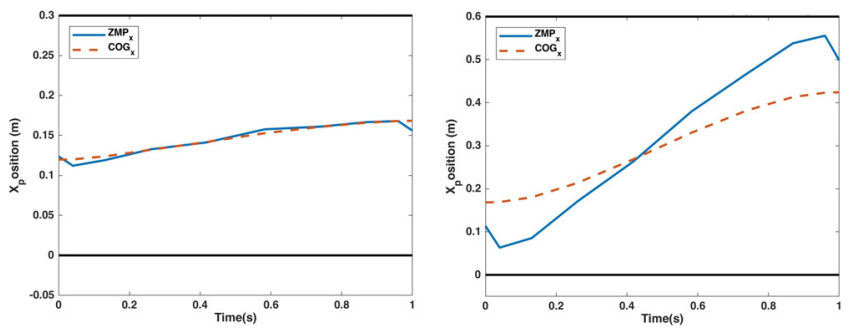

Fig. 7. Example of trajectory of ZMP position. Left: trajectory for single support phase. Right: trajectory for double support phase. Black lines represent the boundary of support base

kinematics scheme.

(5) Apply constrained inverse dynamics to generate the torques that produce the states trajectories defined previously.

Differentiating two times the trajectories of link angles, the three kinematic trajectories are used as input for the constrained inverse dynamics described in Section 2.2, generating control torques for the initial guess.

The initial and final states of the resulting trajectories are presented in Fig. 6. The single support phase brings the robot from figure "a" to figure "b", while the double support phase brings it from figure " $b$ " to figure " $c$ ", which is the mirror of figure " $a$ ". The blue leg is the swinging leg.

4.2 Optimization Process To guarantee stability of balance of walking, the Zero-Moment Point (ZMP) ${ }^{(5)}$ of the model is kept under the base of support during both phases. This is done by defining the allowed boundaries of the ZMP as path constraints in optimization. Details of calculation of ZMP for a model with whole-body dynamics can be found in Sugihara and Fujimoto ${ }^{(23)}$. Figure 7 presents the calculated trajectory for the solution of minimization of friction.

Having the solution of the initial guess, the optimization process was performed following the methodology of Section 3.3. To confirm that our methodology is indeed necessary to achieve solvability, we repeated the optimization removing our techniques one by one and in groups until almost all of them were removed, and checked the results. Removing one or two techniques greatly decreased the precision of optimization (by deteriorating the gain in performance) or doubled computational time or both. The initial guess and the search space tuning were always critical, while scaling was 
Table 2. Computation time for values of discretization

\begin{tabular}{|c|c|}
\hline DISCRETIZATION & COMP TIME \\
\hline $10 \mathrm{pts}$ & $22 \mathrm{~s}$ \\
\hline $20 \mathrm{pts}$ & $5 \mathrm{~min}$ \\
\hline $30 \mathrm{pts}$ & $40 \mathrm{~min}$ \\
\hline $40 \mathrm{pts}$ & overfit \\
\hline
\end{tabular}

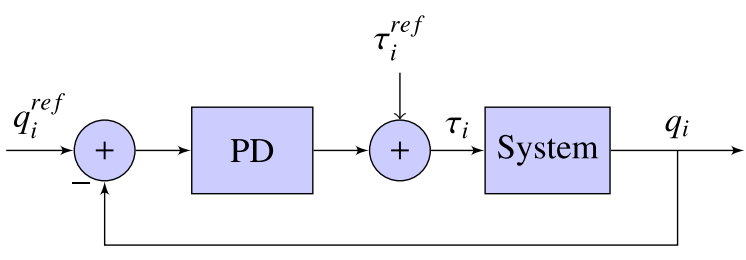

Fig. 8. PD feedback control

critical depending on boundary conditions and cost function. Removing three techniques (scaling, initial guess and search space) made the optimization simply fail and output an error. Removing all techniques was not even possible, since technique described in Section 3.3.1 (elimination of symbolic expressions) cannot be removed without crashing the solver during runtime.

Viable solutions could be found with as little as 10 points of discretization in the numerical solver DIDO. Increasing the discretization did not improve the metrics of energy consumption. Moreover, the computation time increased dramatically with just another 10 or 20 points of discretization, finally coming to overfitting around 40 points. In this situation, the solver cannot find a solution anymore.

With 10 points, solutions were found after only 22 seconds. Even though this is not enough to allow online application, it represents a big improvement from previous attempts at optimizing tasks of robots models with whole-body dynamics while satisfying Pontryagin's Principle. Table 2 shows how the computation time changed with the discretization.

With the parameters of solver tuning (search spaces and equation scaling constants) defined a first time, new solutions can be generated for different parameters of walking with small changes (step length, hip size and duration of task) without repeating the tuning of solver. This suggests the possibility of automatizing the whole process for sets of parameters of walking.

4.3 Simulation The feasibility of all generated solutions was validated using the multibody simulator Simscape Multibody of MATLAB/Simulink software. A simple PD control scheme was used to correct errors of discretization in the angle trajectories (Fig. 8).

In order to evaluate the optimization of gait, the initial guess was also simulated, and its data was compared to the data of the optimal solution. The comparison of performance will be presented in details in Section 5. Figures comparing non-optimal solutions to optimal solutions are presented below. Figure 9 compares the trajectories of torques for their minimization. Figure 10 compares the trajectories of angular velocities of links for the optimization of friction, which affects the velocities directly. In these figures, the indexes 1 to 6 refer to: 1) left ankle, 2) left knee, 3) left hip, 4) right hip, 5) right knee, 6) right ankle.
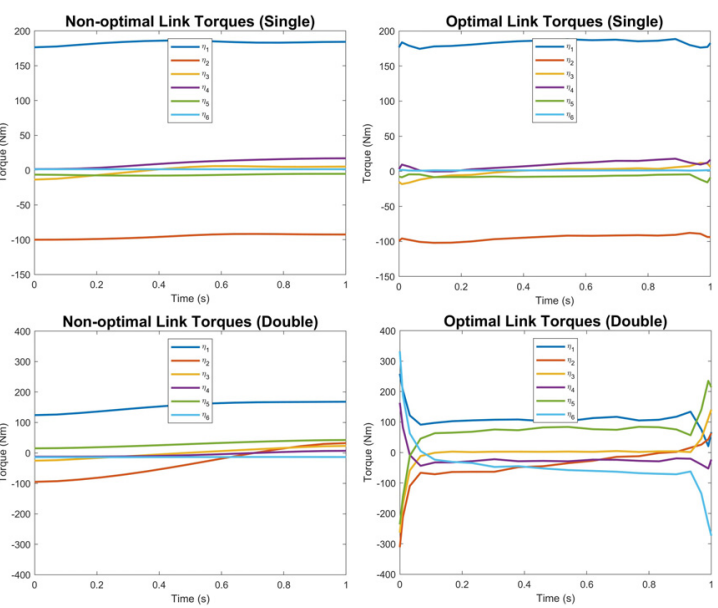

Fig. 9. Trajectory of link torques for optimization of Equation (16). Top: single support phase. Bottom: double support phase. Left: non-optimal solutions. Right: optimal solutions

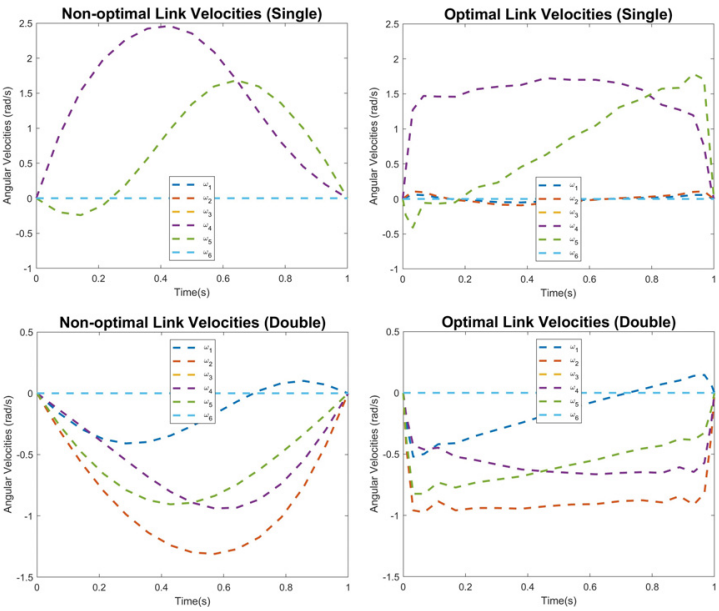

Fig. 10. Trajectory of link angular velocities for optimization of Equation (17). Top: single support phase. Bottom: double support phase. Left: non-optimal solutions. Right: optimal solutions

\section{Performance Analysis}

5.1 Optimization Results The direct evaluation of success of optimization is the calculation of the cost function for both optimal and non-optimal solutions (Equation (16) for Torque, Equation (17) for Friction and Equation (18) for Energy). The results are given in Table 3. To provide further insights, all three cost functions are calculated for each of the three optimizations performed. As can be observed in the tables, every optimal solution has a better performance than the non-optimal solution, confirming the success and overall accuracy of optimization.

A more in-depth evaluation of accuracy of optimization is the confirmation of optimality between optimal solutions. The expected behavior for every type of optimization is that its particular metric of optimality should be better than the same metric in other optimizations (e.g. the minimization of torques should be better for "T sol" than for other optimizations). This is confirmed in most optimizations except 
Table 3. Value of cost functions for all non-optimal and optimal solutions. $\mathbf{N}$ sol is the non-optimal solution, $\mathbf{T}$ sol is the solution of torque minimization, $\mathbf{F}$ sol is the solution of friction minimization and $\mathbf{P}$ sol is the solution of power minimization

\begin{tabular}{|c|c|c|c|c|c|c|}
\hline & \multicolumn{3}{|c|}{ Single support phase } & \multicolumn{3}{c|}{ Double support phase } \\
\hline Cost Type & $\begin{array}{c}\text { Torque } \\
(\mathrm{Nm})^{2} s\end{array}$ & $\begin{array}{c}\text { Friction } \\
10^{-4}(\mathrm{Nm})^{2} s\end{array}$ & $\begin{array}{c}\text { Power } \\
W s\end{array}$ & $\begin{array}{c}\text { Torque } \\
(\mathrm{Nm})^{2} s\end{array}$ & $\begin{array}{c}\text { Friction } \\
10^{-4}(\mathrm{Nm})^{2} s\end{array}$ & $\begin{array}{c}\text { Power } \\
W s\end{array}$ \\
\hline \hline N sol & $\mathbf{2 8 3 . 0}$ & $\mathbf{2 . 7 1 2 2}$ & $\mathbf{8 . 3 9 0 5}$ & $\mathbf{3 1 7 . 7}$ & $\mathbf{1 . 2 8 1 3}$ & $\mathbf{- 8 . 2 1 6 1}$ \\
\hline \hline T sol & $\mathbf{2 3 4 . 2}$ & 2.1651 & 8.3684 & $\mathbf{2 8 0 . 9}$ & 1.1025 & -8.2592 \\
\hline \hline F sol & 234.4 & $\mathbf{2 . 1 4 5 0}$ & 8.3726 & 338.5 & $\mathbf{1 . 0 9 7 5}$ & -8.2498 \\
\hline \hline P sol & 280.4 & 2.6913 & $\mathbf{8 . 3 7 4 0}$ & 284.2 & 1.2932 & $\mathbf{- 8 . 3 0 1 0}$ \\
\hline
\end{tabular}

Table 4. Percentage decrease of value of cost function from non-optimal to optimal solutions. Decrease calculated for each solution using only their own cost function

\begin{tabular}{|c|c|c|}
\hline & Single Phase & Double Phase \\
\hline \hline T sol & $17.2 \%$ & $11.6 \%$ \\
\hline \hline F sol & $20.9 \%$ & $14.3 \%$ \\
\hline \hline P sol & $0.197 \%$ & $1.02 \%$ \\
\hline
\end{tabular}

for minimization of power in the joints in the single support phase, which is very close to the most optimal in each case. This anomalous behavior is concluded to be due to the nonconvexity of the optimization and its subsequent inaccuracy. Further studies are being made to guarantee a proper and accurate optimization of power and other non-convex optimizations.

Table 4 shows the percentage decrease of value of cost function from non-optimal to optimal solutions, calculated only for the own cost function of each minimization. The first two minimizations (torque and friction) showed significant improvements of around 15-20\%, but the minimization of power showed very minor improvement.

The result of minimization of torques applied in the joints was successful in terms of cost function value. Investigating details of the solution in Fig. 9, we see how the torques applied in the joints were minimized (together with a smaller term minimizing the angular velocities) by applying big torques for a very short time in the beginning and end of solution to accelerate the links faster and apply smaller torques during most of the task. Since minimization of torques usually are performed to achieve smooth controls (e.g. lesser peaks), and not necessarily average smaller torques, the results suggest that smoothness is best served by another formulation in the cost function instead of square of torques.

The minimization of friction was also successful in terms of cost function. Analysing Fig. 10, in both phases (single and double support phase) the optimal solution shows a decrease of peak in the angular velocities in respect to the nonoptimal solution. Since the task is being performed in the same time, this is compensated by a bigger acceleration. The consequence is a more constant velocity in the optimal solution. This fact confirms that the optimization is working to reduce friction, since keeping the velocity smaller and more constant is a natural consequence of minimizing the square of friction.

As mentioned earlier, the minimization of applied power was negligible, amounting to $1 \%$ at most. Checking how the other two minimizations performed in this same metric, the same range of improvement was observed. While the results are not conclusive due to the non-convexity of minimizing non-squared power, they suggest a limit in decreasing of energy consumption modeling only losses by friction. Further studies will be performed to enforce the validity of non-convex optimization, while best modeling of losses are concluded to be necessary both for mechanical losses (e.g. impact with the floor) and electrical losses (switching and resistance losses in circuits and motor drives).

A more qualitative evaluation of optimization is possible checking the visuals of all the simulations. It was noted how the heel of the swinging leg is briefly lifted in the beginning of the swinging phase, indicating a simplified reproduction of the behavior of human gait in the model gait. A human gait lifts the swinging leg and then sweeps it, taking advantage of its dynamics and gravity to move the leg without fully actuating it. The non-optimal solution does not present this behavior because the control is simply "carrying" the weight of the leg in a smooth and symmetrical trajectory for position and velocity. What is notable, however, is how the optimization reproduced this human behavior in a gait with a physical structure and parameters that would not favour it. While human gait lifts the leg and sweeps the ground, it is at the same time lifting the height of the hip. This is done to allow the leg to swing freely, without hitting the ground. But our gait is designed with constant hip height. The fact that the optimization still reproduced slightly this strategy suggests a natural energetic optimality in it, and that if the gait was designed with the same inverted pendular movement of hip heigth, the lifting of the swinging leg would be more accentuated and more exploited by the optimization.

\section{Summary and Future Works}

This work presented a novel methodology developed to consistently solve the optimization of robotic bipedal walking as a nonlinear multi-degree-of-freedom whole-body dynamical problem. Common simplifications in literature were avoided: the simplification of dynamical models and the use of direct methods of optimization that do not estimate the costates of Pontryagin's Principle. The methodology is based on numerical techniques that exploit the evaluation of analytical expressions, decrease the numerical conditioning of differential equations, decrease the sensitivity of the optimization to the costates and make use of problem-specific non-optimal solutions to properly initialize the optimization and tune the solver. These techniques address inherent challenges of optimization and can be applied independently of method/solver.

To showcase the achieved consistency in solvability, we realized the pseudospectral optimization of robotic bipedal walking using the whole-body dynamics and three different objectives of optimization: minimization of torques, friction and power applied in the joints. Without the techniques, the method/solver used could not successfuly perform the optimizations. The successful runs took about $22 \mathrm{~s}$.

For future works, we plan to extend the planar model to a full 3D model, use more dynamic balancing criteria for generating the initial guess, validate our methodology with different optimal control methods/solvers and compare their performances. 


\section{References}

( 1 ) G.H.Z. Liu, M.Z.Q. Chen, and Y. Chen: "When joggers meet robots: the past, present, and future of research on humanoid robots", Bio-Design and Manufacturing, Vol.2, No.2, pp.108-118 (2019)

( 2 ) E. Krotkov, D. Hackett, L. Jackel, M. Perschbacher, J. Pippine, J. Strauss, G. Pratt, and C. Orlowski: "The DARPA Robotics Challenge Finals: Results and Perspectives", Journal of Field Robotics, Vol.34, No.2, pp.229-240 (2017)

( 3 ) T. Boer: "Foot placement in robotic bipedal locomotion", Ph.D. thesis, Delft University, The Netherlands (2012)

( 4 ) S. Kajita, F. Kanehiro, K. Kaneko, K. Fujiwara, K. Harada, K. Yokoi, and H. Hirukawa: "Resolved momentum control: humanoid motion planning based on the linear and angular momentum", Proc. IEEE/RSJ International Conference on Intelligent Robots and Systems (IROS 2003), Vol.2, pp.1644-1650 (2003)

( 5 ) M. Vukobratovic and B. Borovac: "Zero-moment Point-Thirty Five Years of its Life", International Journal of Humanoid Robotics, Vol.1, No.1, pp.157-173 (2004)

( 6 ) J.W. Grizzle and C. Chevallereau: "Virtual Constraints and Hybrid Zero Dynamics for Realizing Underactuated Bipedal Locomotion", Computing Research Depository, http://arxiv.org/abs/1706.01127 (2017)

( 7 ) M. Wisse, G. Feliksdal, J. Frankenhuyzen, and B. Moyer: "Passive-based walking robot-Denise, a simple, efficient, and lightweight biped", IEEE Robotics and Automation Magazine, Vol.14, No.2, pp.52-62 (2007)

( 8 ) I.M. Ross: “A primer on Pontryagin's principle in optimal control”, Collegiate Publishers (2009)

( 9 ) D.E. Kirk: "Optimal Control Theory: An Introduction", Dover Publications (2004)

(10) D.S. Naidu: "Optimal Control Systems", CRC Press (2002)

(11) R.M. Carnier and Y. Fujimoto: "Optimization and Comparison of Human and Avian Robotic Walking", Proc. 19th IEEE/ASME International Conference on Advanced Intelligent Mechatronics (AIM), Boston, USA, pp.85-90 (2020)

(12) I.M. Ross and F. Fahroo: "A Perspective on Methods for Trajectory Optimization", Proc. AIAA/AAS Astrodynamics Specialist Conference and Exhibit, AIAA 2002-4727 (2002)

(13) O.V. Stryk and R. Bulirsch: "Direct and Indirect Methods for Trajectory Optimization", Annals of Operations Research., Vol.37, No.1, pp.357-373 (1992)

(14) P. Grüne: "Numerical Methods for Nonlinear Optimal Control Problems", In: J. Baillieul, T. Samad: "Encyclopedia of Systems and Control", pp.1-10, Springer (2014)

(15) A. Rao: "A Survey of Numerical Methods for Optimal Control", Advances in the Astronautical Sciences, Vol.135, No.1, pp.497-528 (2010)

(16) I.M. Ross: "A historical introduction to the covector mapping principle", $A d$ vances in the Astronautical Sciences: Astrodynamics, Vol.122, pp.05-332 (2005)

(17) F. Fahroo and I.M. Ross: "Costate Estimation by a Legendre Pseudospectral Method", Journal of Guidance, Control, and Dynamics, Vol.24, No.2, pp.270-277 (2001)

(18) S. Lengagne, J. Vaillant, E. Yoshida, and A. Kheddar: "Generation of wholebody optimal dynamic multi-contact motions", The International Journal of Robotics Research, Vol.32, No.9-10, pp.1104-1119 (2013)

(19) K.H. Koch, K. Mombaur, and P. Soueres: "Optimization-based walking generation for humanoids", IFAC Proceedings Volumes, Vol.45, No.22, pp.498504 (2012)

(20) D. Clever, M. Harant, K. Mombaur, M. Naveau, O. Stasse, and D. Endres: "Cocomopl: A novel approach for humanoid walking generation combining optimal control, movement primitives and learning and its transfer to the real robot HRP-2", IEEE Robotics and Automation Letters, Vol.2, No.2, pp.977984 (2017)
(21) Y. Hu and K. Mombaur: "Bio-Inspired Optimal Control Framework to Generate Walking Motions for the Humanoid Robot iCub Using Whole Body Models", Applied Sciences, Vol.8, No.2, p.278 (2018)

(22) M. Rostami and G. Bessonnet: "Impactless sagittal gait of a biped robot during the single support phase", Proc. 1998 IEEE International Conference on Robotics and Automation, Vol.2, pp.1385-1391 (1998)

(23) T. Sugihara and Y. Fujimoto: "Dynamic Analysis: Equations of Motion", In: A. Goswami, P. Vadakkepat: "Humanoid Robotics: A Reference", pp.723754, Springer (2018)

(24) J.C.A. Barata and M.S. Hussein: "The Moore-Penrose Pseudoinverse: A Tutorial Review of the Theory", Brazilian Journal of Physics, Vol.42, No.1-2, pp.146-165 (2011)

(25) R.M. Carnier and Y. Fujimoto: "Precise Optimization of Robotic Bipedal Walking Using Hamiltonian Dynamics", Proc. 29th IEEE International Symposium on Industrial Electronics (ISIE), Delft, Netherlands, pp.567-572 (2020)

(26) I.M. Ross: “A Beginner's guide to DIDO: A MATLAB application package for solving optimal control problems", Elissar Global (2007)

(27) I.M. Ross and F. Fahroo: "Legendre pseudospectral approximations of optimal control problems", Lecture Notes in Control and Information Sciences, Vol.295, pp.327-342, Springer-Verlag (2003)

(28) R.M. Carnier and Y. Fujimoto: "Energy-efficient Optimal Control of Robotic Leg by Indirect Methods", Proc. 43rd Annual Conference of the IEEE Industrial Electronics Society (IECON 2017), pp.6721-6726 (2017)

(29) R.M. Carnier and Y. Fujimoto: "Pseudospectral Optimization of Planar Robotic Walking using Whole-body Dynamics", 6th IEEJ International Workshop on Sensing, Actuation, Motion Control and Optimization (SAMCON), Japan (2020)

(30) I.M. Ross, Q. Gong, M. Karpenko, and R.J. Proulx: "Scaling and Balancing for High-Performance Computation of Optimal Controls", Journal of Guidance, Control, and Dynamics, Vol.41, No.10, pp.2086-2097 (2018)

(31) I.M. Ross: "Scaling and balancing optimal control problems: A must-read document for any serious user of DIDO”, Elissar Global (2016)

Rodrigo Matos Carnier (Non-member) received the B.E. degree in

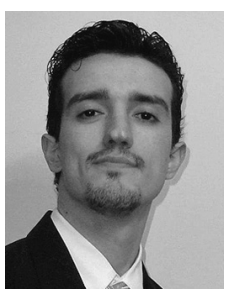
mechatronics engineering from University of Sao Paulo, Sao Paulo, Brazil, in 2013, and the M.E. degree in electrical and computer engineering from Yokohama National University, Yokohama, Japan, in 2017, where he is currently a Ph.D. candidate. His research interests include biped locomotion, motion control, robotics and optimal control.

Yasutaka Fujimoto (Senior Member) received the B.E., M.E., and

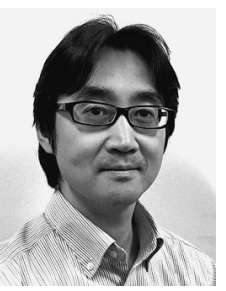
$\mathrm{Ph} . \mathrm{D}$. degrees in electrical and computer engineering from Yokohama National University, Yokohama, Japan, in 1993, 1995, and 1998, respectively. In 1998, he joined the Department of Electrical Engineering, Keio University, Yokohama, Japan. Since 1999, he has been with the Department of Electrical and Computer Engineering, Yokohama National University, where he is currently a Professor. His research interests include actuators, robotics, manufacturing automation, and motion control. Dr. Fujimoto is an Associate Editor of the IEEJ-JIA and IEEE-TIE. 